\title{
Apelin: A potential novel serum biomarker for early detection of diabetic nephropathy in patients with type 2 diabetes
}

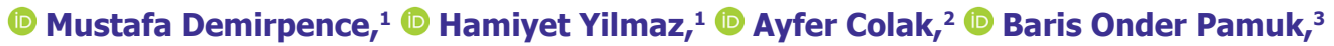 \\ (D) Inanc Karakoyun, ${ }^{2}$ (D) Banu Basok ${ }^{2}$ \\ ${ }^{1}$ Department of Endocrinology and Metabolism, Tepecik Training and Research Hospital, Izmir, Turkey \\ 2Department of Clinical Biochemistry, Tepecik Training and Research Hospital, Izmir, Turkey \\ ${ }^{3}$ Department of Endocrinology and Metabolism, Izmir Katip Celebi University, Ataturk Training and Research Hospital, Izmir, Turkey
}

\begin{abstract}
OBJECTIVE: Diabetic nephropathy (DN) is the major cause of chronic renal failure, and proteinuria is an independent risk factor for the end stage renal disease. The random urine protein:creatinine ratio ( $P: C$ ratio) can accurately predict the amount of 24-hour urinary protein excretion. Apelin is thought to be associated with endothelial dysfunction, angiogenesis, and inflammation. This study investigated the apelin concentration and its association with the urine P:C ratio, and metabolic parameters in subjects with and without type 2 diabetes mellitus (T2D).

METHODS: This study involved 86 subjects: 56 with newly diagnosed and untreated T2D and 30 non-diabetic controls. All subjects underwent a complete clinical examination that included anthropometric and laboratory measurements.

RESULTS: Twenty-four males and sixty-two females participated in this study, and their mean age was $52.27 \pm 11.34$ years. There were no differences in age, thyrotropin-stimulating hormone (TSH), creatinine clearance, and apelin levels between groups. As expected, fasting plasma glucose, weight, body mass index, and HbA1C were higher in T2D subjects ( $\mathrm{p}=0.001$, $p=0.02, p=0.03$, and $p=0.001$, respectively). Although apelin levels were higher in the control group, the differences were not statistically significant $(p=0.93)$. The $P: C$ ratio levels were lower in the control group, and the differences were statistically significant $(p=0.006)$. A Spearman correlation analysis revealed that serum apelin levels were not correlated with the urine P:C ratio. CONCLUSION: Our study demonstrates that T2D is associated with decreased serum apelin levels and increased urine $P: C$ ratios compared to those in non-diabetic subjects. This association may depend on impaired glucose homeostasis. Our results show that the serum apelin levels were not correlated with the urine protein:creatinine ratio and provide further evidence regarding the relationship between apelin and DN.
\end{abstract}

Keywords: Apelin; diabetic nephropathy; urine protein: creatinine ratio.

Cite this article as: Demirpence M, Yilmaz H, Colak A, Pamuk BO, Karakoyun I, Basok B. Apelin: A potential novel serum biomarker for early detection of diabetic nephropathy in patients with type 2 diabetes. North Clin Istanb 2019;6(2):151-155.

$\mathrm{D}$ iabetic nephropathy (DN) is a common microvascular complication of diabetes that is characterized by glomerular, tubular, and tubulointerstitial injury resulting from hyperglycemic conditions [1]. The earliest pathological features of DN include glomerular hypertrophy and glomerular basement membrane thickening [1]. Disease progression can lead to clinical manifestations including albuminuria, hypertension, and a re-

Received: June 03, 2017 Accepted: February 19, 2018 Online: August 14, 2018

Correspondence: Dr. Mustafa DEMIRPENCE. Tepecik Egitim ve Arastirma Hastanesi, Endokrinoloji ve Metabolizma Hastaliklari Klinigi, Izmir, Turkey.

Tel: +90 5055252337 e-mail: dr.mustafa.demirpence@gmail.com

(c) Copyright 2019 by Istanbul Provincial Directorate of Health - Available online at www.northclinist.com 
duced glomerular filtration rate. Previous experimental and clinical studies have demonstrated significant roles of various inflammatory molecules in the setting of diabetic nephropathy, including acute phase reactants, inflammatory cytokines, adhesion molecules, and chemokines [2].

Proteinuria is an independent risk factor for renal diseases. Thus, accurate identification and quantification of proteinuria are critical for the diagnosis and management of chronic renal diseases. Proteinuria is associated with an increased risk of progressive renal failure, cardiovascular diseases, and death. Additionally, increased proteinuria has both diagnostic and prognostic values in the detection and confirmation of renal diseases [3].

The use of timed urine collections (usually performed over 24 hours) is currently considered the gold standard for proteinuria quantification. However, there are major limitations associated with this method, such as the time requirement and errors in sample collection. The estimation of total protein in a spot urine sample avoids 24-hour urine collection but is influenced by body hydration. This variation can be eliminated by factoring the spot urine total protein concentration by the urine creatinine concentration, which is reported as the urine protein to creatinine ratio ( $\mathrm{P}: \mathrm{C}$ ratio) [4]. Therefore, random urine sampling to evaluate the $\mathrm{P}: \mathrm{C}$ ratio could be more acceptable and less time consuming [5]. The random urine $\mathrm{P}: \mathrm{C}$ ratio predicts the amount of 24-hour urinary protein excretion with high accuracy. Thus, the $P: C$ ratio can be used as a faster diagnostic substitute for 24-hour urinary protein estimation [6].

Apelin is a newly discovered adipocytokine [7] that is widely expressed in organs such as the kidney, heart, lung, adipose tissue, liver, endothelium, and human plasma. Apelin also leads to endothelial cell proliferation and angiogenesis [8]. Apelin and its receptor APJ have pleiotropic effects in mice and humans and have a protective role in cardiovascular diseases by partially inhibiting oxidative stress. Oxidative stress has an important role in the development of DN [9]. Thus, apelin may have a role in DN. In this study, we investigated the apelin concentration and its association with the urine protein to creatinine ratio, and other metabolic parameters in a cohort of individuals with and without type 2 diabetes mellitus (T2D).

\section{MATERIALS AND METHODS}

This study was designed as a case-control study. We recruited 86 subjects: 56 patients with T2D and 30 nondiabetic controls were enrolled from the endocrinology and metabolism disease outpatient clinics of Tepecik
Training and Research Hospital, Izmir, Turkey. The study population consisted of 56 patients with newly diagnosed and untreated T2D and 30 non-diabetic controls matched for age and sex. All subjects received a health assessment that included a clinical examination, anthropometric measurements, and laboratory tests. The subjects were free from the following chronic diseases: hypercortisolism, chronic renal disease, chronic liver disease, history of cancer, and cardiovascular disease.

\section{Laboratory Assessments}

Blood samples were collected from all subjects after fasting by venous puncture using Vacationer (BD Diagnostics, Plymouth, UK) tubes with ethylene diamine tetra acetic acid and no anticoagulants were used for the biochemical determinations. The serum samples were obtained after the blood samples were centrifuged at 4,000 rpm for 10 minutes. The resultant serum samples were aliquoted into polypropylene tubes and stored at $-80^{\circ} \mathrm{C}$ until the biochemical analysis was conducted.

The fasting plasma glucose (FPG) concentrations were measured using the hexokinase method with an Olympus AU-2700 analyzer. The levels of blood triglycerides (TG), total cholesterol, and high-density lipoprotein (HDL) cholesterol were measured by an enzymatic method with the Olympus AU-2700 analyzer using reagents from Olympus Diagnostics Gmbh (Hamburg, Germany). The low-density lipoprotein (LDL)-cholesterol was calculated using the Friedewald equation. Other routine biochemical measurements were performed on the same day using enzymatic methods and the Olympus AU 2700 auto-analyzer (Olympus, Tokyo, Japan).

The thyrotropin-stimulating hormone (TSH) level was measured the same day with the electrochemiluminescence method (ECLIA) by using a Cobas E 411 (Roche Diagnostics). The glycated hemoglobin (HbA1c) analysis was performed by high-pressure liquid chromatography on a Primus instrument. The complete blood count analysis was performed in an automated complete blood count device (Beckman Coulter).

The urine $P: C$ ratio was determined using spot urine specimens. The concentration of total protein in urine was measured by a biuret colorimetric assay (Cobas Integra Analyzer, F Hoffman-La Roche, Basel, Switzerland). The urine creatinine level was measured by a modified Jaffe test (Hitachi 7170 auto-analyzer, Hitachi, Tokyo, Japan). The $\mathrm{P}: \mathrm{C}$ ratio was calculated for both samples by dividing the urinary proteinuria $(\mathrm{mg} / \mathrm{dL})$ by creatinine $(\mathrm{mg} / \mathrm{dL})$.

The estimated creatinine clearance $(\mathrm{CCr})$ was derived 
using the method of Cockcroft and Goult. The CCr was calculated using the Cockcroft-Goult equation: [(140 age $) \times$ total body weight $] /($ serum creatinine $(\mathrm{mg} / \mathrm{dL}) \times$ 72) $(\times 0.85$ for females $)[10]$.

The serum apelin concentrations were measured using an enzyme-linked immunosorbent assay kit according to the manufacturer's instructions (Apelin; Catalog No.: 201-12-2015, Sunred Biological Technology, Shanghai, China). The intra-assay and inter-assay coefficients of variations were $<10 \%$ and $<12 \%$ for apelin, respectively.

\section{Anthropometric Measurements}

The height of each subject was measured to the nearest $0.1 \mathrm{~cm}$ using a stadiometer. The body weight of each subject was measured using a digital weighing scale (ADE, Germany, Type MZ 10023). The body mass index (BMI) was measured in all study subjects. The BMI was calculated as the ratio between weight and height squared in $\mathrm{kg} / \mathrm{m}^{2}$. The blood pressure was measured with the person in a seated position after a 5-min rest using an Omron M3 HEM-7131 electronic, auscultatory blood pressure reading machine. The first reading was discarded and then the mean of the next three consecutive readings was used.

\section{Ethics Statement}

This study was approved by the medical ethics committee of the Tepecik Training and Research Hospital. All participants provided written informed consent to participate in this study, as approved by the Ethics Committee of the Izmir Tepecik Training and Research Hospital (Date: August 18, 2016; Meeting Number :29; Decision: 2 ) and in accordance with the Declaration of Helsinki.

\section{Statistical Analysis}

All data were analyzed using the Statistical Package for Social Sciences software version 15.0 (SSPS, Inc., Chicago, Illinois, USA). An alpha level of 0.05 was used to determine statistically significant differences. Shapiro-Wilk tests were performed to assess the normal distribution of continuous variables. The data were reported as the mean \pm standard deviation for normally distributed variables. The variables with non-normal distribution were reported as medians with minimum and maximum values. The categorical variables were evaluated using Chi square tests. The normally distributed values of continuous variables between the case and control groups were evaluated with an independent $t$-test. All non-normally distributed values of continuous vari-
TABLE 1. Clinical characteristics of participants

\begin{tabular}{lccc} 
& Type 2 & Non-Type 2 & $p$ \\
& diabetes $(n=56)$ & diabetes $(n=30)$ & \\
\hline Age (mean \pm SD) & $53.85 \pm 11.0$ & $49.33 \pm 11.57$ & 0.92 \\
Hypertension, Yes/No & $25 / 31$ & $10 / 20$ & 0.299 \\
Gender, M/F & $15 / 41$ & $9 / 21$ & 0.751 \\
\hline
\end{tabular}

SD: Standard deviation; $\mathrm{p}<0.05$ was considered statistically significant.

ables between the case and control groups were evaluated with the Mann-Whitney $U$ test. In this study, $p<0.05$ was considered statistically significant.

\section{RESULTS}

There were 24 males and 62 females in the study, and their mean age was $52.27 \pm 11.34$ years. The data indicate there were no differences in age, BMI, FPG, lipid parameters, $\mathrm{HbA} 1 \mathrm{c}$, and apelin levels between genders. The gender distribution was similar for the T2D (male/ female: 15/41) and control (without T2D; male/female: $9 / 21)$ groups $(p=0.751)$. The hypertension ratio distribution was similar between the T2D (Yes/No: 25/31) and control (without T2D; Yes/No: 10/20) groups $(p=0.29)$. The mean age (years) was similar between the T2D $(53.85 \pm 11.0)$ and control $(49.33 \pm 11.57)$ groups $(\mathrm{p}=0.92)$ (Table 1$)$.

The fasting glucose and $\mathrm{HbA} 1 \mathrm{c}$ were higher in T2D subjects $(\mathrm{p}=0.001$ and $\mathrm{p}=0.001$, respectively).

Although TSH, LDL, total cholesterol, creatinine clearance levels, systolic blood pressure, and diastolic blood pressure were not significantly different between the two groups $(p=0.07, p=0.107, p=0.363, p=0.23, p=0.13$, and $\mathrm{p}=0.141$, respectively), the HDL values were lower in the T2D subjects than in the control subjects $(p=0.002)$. Additionally, the TG levels were higher in the T2D subjects than in the control subjects $(p=0.005)$. The apelin levels were higher in the control group, but the differences were not statistically significant $(p=0.93)$. The $P: C$ ratio levels were higher in the control group, and the differences were statistically significant $(p=0.006)$ (Table 2$)$.

The Spearman correlation analysis revealed that serum apelin levels were not correlated with urine $P: C$ ratio, BMI, body weight, fasting glucose, TSH, HbA1c, lipid parameters, and creatinine clearance. 
TABLE 2. Comparison of anthropometric and metabolic parameters between subjects with and without type 2 diabetes

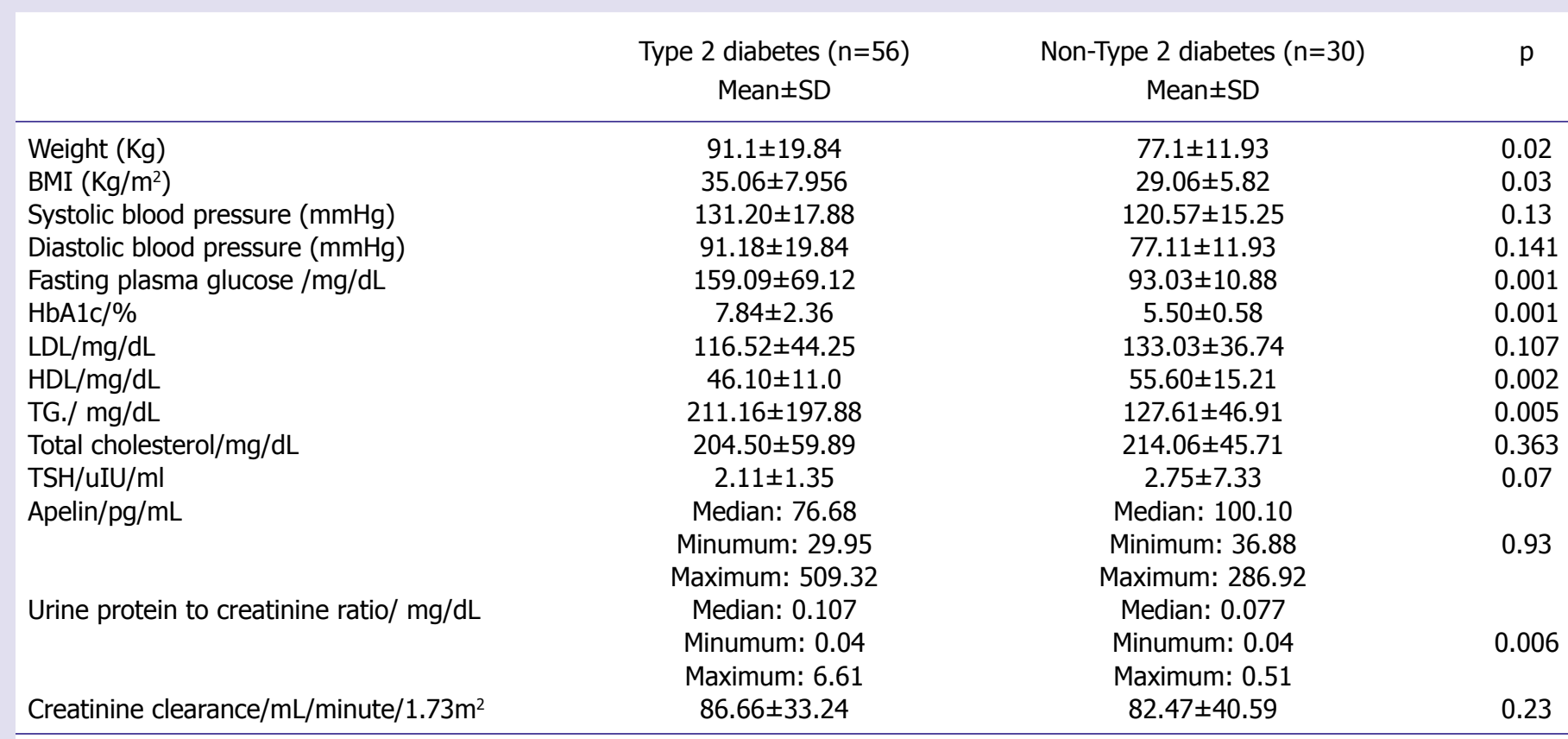

SD: Standard deviation; BMI: Body mass index; HbA1c: Glycated hemoglobin; HDL: High-density lipoprotein cholesterol; LDL: Low-density lipoprotein cholesterol; TSH: Thyrotropin-stimulating hormone; TG: Triglycerides; $\mathrm{p}<0.05$ was considered statistically significant.

\section{DISCUSSION}

Type 2 diabetes represents a major health problem and is associated with an increased risk for both all-cause and cardiovascular disease mortality. DN is currently the leading cause of end-stage renal disease. It is also a significant long-term complication in terms of morbidity and mortality for diabetic patients [11]. The diagnostic markers used to detect DN at an early stage are important because early intervention can slow the loss of renal function and improve patient outcomes. The aim of this study was to evaluate the diagnostic value of serum apelin as a biomarker for the early detection of nephropathy in T2D patients.

In the present study, the levels of FPG and $\mathrm{HbA1c}$ in the diabetic group were significantly increased and that the urine P:C ratio was higher than that in the control group. These findings are consistent with those from previous studies suggesting that hyperglycemia drives DN development [12].

The results of this study demonstrated that apelin serum levels in non-diabetic subjects tend to be slightly higher than those in the individuals with T2DM, but the difference was not statistically significant. There are conflicting results about apelin levels in type 2 diabetes. While it was found to be increased in some studies [13, 14], it was reported to be decreased in others [15]. Lower apelin levels in type 2 diabetes may be explained by that apelin regulates insulin sensitivity, stimulates glucose utilization, and enhances brown adipogenesis in different tissues associated with diabetes [16].

Apelin has a close relationship with metabolism. Previous studies found that apelin levels in serum were increased and associated with glucose homeostasis and BMI in T2D cases $[17,18]$. However, other studies reported that obese patients with T2D had significantly higher apelin levels than non-diabetic obese subject. This finding confirms that increased apelin levels are directly associated with the presence of diabetes rather than obesity itself [18]. Also, as observed by Boucher et al., while plasma apelin concentrations were increased significantly in different mouse models of obesity, there was no increase in apelin plasma concentrations in the non-hyperinsulinemic obese mouse. These findings suggest that insulin could influence serum apelin levels [19].

In the present study, the mean serum levels of TG were higher in the diabetic group than in the control group. Conversely, the mean serum level of HDL was sig- 
nificantly lower in the diabetic groups than in the control group. These results are in agreement with the study by Bonnet and Cooper [20]. The authors reported that DN is associated with altered lipid metabolism characterized by elevated TG-rich lipoproteins in the early stages of renal disease. These results are also in agreement with the study by Jacobs et al. [21], which concluded that the prevalence of dyslipidemia was higher in type 2 diabetic patients than in healthy subjects.

In our study, we did not find any relationship between apelin and urine $\mathrm{P}: \mathrm{C}$ ratio in patients with T2D. Although in animal studies apelin was shown to induce angiogenesis, increased glomerular permeability, and albuminuria leading to $\mathrm{DN}$, the association between apelin and development of microvascular complications in type 2 diabetes was not observed in many clinical studies [2224]. In one study, plasma apelin level was not changed in $\mathrm{DR}$, but, there was a positive correlation between apelin levels and urinary albumin/creatinine ratio [24]. This finding suggests that there may be an inverse association between DN and apelin under physiological or pathological conditions.

Our study has several limitations. The patient sample size was small due to the narrow selection criteria. As a result, our data may not be representative of all subjects with T2D.

Additional experiments are needed to determine whether there is cooperation between other angiogenic factors and apelin. The effect of apelin on proliferation, migration, and vascular endothelial function and the underlying mechanisms also need to be studied. Thus, larger studies in subjects with and without vascular complications or $\mathrm{DN}$ are required to investigate the role of apelin in T2D.

Conflict of Interest: The authors declare that there are no conflicts of interest regarding the publication of this manuscript.

Financial Disclosure: The authors declared that this study has received no financial support.

Authorship Contributions: Concept - MD; Design - MD, HY, BOP; Supervision - MD, AC, IK; Data collection and/or processing - MD, $\mathrm{BB}, \mathrm{HY}$; Analysis and/or interpretation - MD, HY, BOP, AC; Writing MD, HY, BOP; Critical review - MD, HY, BOP, AC, IK, BB.

\section{REFERENCES}

1. Cooper ME. Interaction of metabolic and haemodynamic factors in mediating experimental diabetic nephropathy. Diabetologia 2001;44:195772. [CrossRef]

2. Navarro-González JF, Mora-Fernández C. The role of inflammatory cytokines in diabetic nephropathy. J Am Soc Nephrol 2008;19:433-42.
3. Methven S, MacGregor MS, Traynor JP, O'Reilly DS, Deighan CJ. Assessing proteinuria in chronic kidney disease: protein-creatinine ratio versus albumin-creatinine ratio. Nephrol Dial Transplant 2010;25:2991-6. [CrossRef]

4. Stevens LA, Levey AS. Current status and future perspectives for CKD testing. Am J Kidney Dis 2009;53:S17-26. [CrossRef]

5. Cirillo M. Evaluation of glomerular filtration rate and of albuminuria/ proteinuria. J Nephrol 2010;23:125-32.

6. Biradar SB, Kallaganad GS, Rangappa M, Kashinakunti SV, Retnakaran $\mathrm{R}$. Correlation of spot urine protein-creatinine ratio with 24-hour urinary protein in type 2 diabetes mellitus patients: A cross sectional study. J Res Med Sci 2011;16:634-9.

7. Tatemoto K, Hosoya M, Habata Y, Fujii R, Kakegawa T, Zou MX, et al. Isolation and characterization of a novel endogenous peptide ligand for the human APJ receptor. Biochem Biophys Res Commun 1998;251:471-6. [CrossRef]

8. Kasai A, Shintani N, Oda M, Kakuda M, Hashimoto H, Matsuda T, et al. Apelin is a novel angiogenic factor in retinal endothelial cells. Biochem Biophys Res Commun 2004;325:395-400. [CrossRef]

9. Day RT, Cavaglieri RC, Feliers D. Apelin retards the progression of diabetic nephropathy. Am J Physiol Renal Physiol 2013;304:F788-800.

10. Cockcroft DW, Gault MH. Prediction of creatinine clearance from serum creatinine. Nephron 1976;16:31-41. [CrossRef]

11. Nichols GA, Vupputuri S, Lau H. Medical care costs associated with progression of diabetic nephropathy. Diabetes Care 2011;34:2374-8.

12. Sun YM, Su Y, Li J, Wang LF. Recent advances in understanding the biochemical and molecular mechanism of diabetic nephropathy. Biochem Biophys Res Commun 2013;433:359-61. [CrossRef]

13. Habchi M, Duvillard L, Cottet V, Brindisi MC, Bouillet B, Beacco M, et al. Circulating apelin is increased in patients with type 1 or type $2 \mathrm{di}$ abetes and is associated with better glycaemic control. Clin Endocrinol (Oxf) 2014;81:696-701. [CrossRef]

14. Li L, Yang G, Li Q, Tang Y, Yang M, Yang $H$, et al. Changes and relations of circulating visfatin, apelin, and resistin levels in normal, impaired glucose tolerance, and type 2 diabetic subjects. Exp Clin Endocrinol Diabetes 2006;114:544-8. [CrossRef]

15. Erdem G, Dogru T, Tasci I, Sonmez A, Tapan S. Low plasma apelin levels in newly diagnosed type 2 diabetes mellitus. Exp Clin Endocrinol Diabetes 2008;116:289-92. [CrossRef]

16. $\mathrm{Hu} \mathrm{H}, \mathrm{He} \mathrm{L}, \mathrm{Li} \mathrm{L}$, Chen L. Apelin/APJ system as a therapeutic target in diabetes and its complications. Mol Genet Metab 2016;119:20-7.

17. Yu S, Zhang Y, Li MZ, Xu H, Wang Q, Song J, et al. Chemerin and apelin are positively correlated with inflammation in obese type 2 diabetic patients. Chin Med J (Engl) 2012;125:3440-4.

18. Cavallo MG, Sentinelli F, Barchetta I, Costantino C, Incani M, Perra $\mathrm{L}$, et al. Altered glucose homeostasis is associated with increased serum apelin levels in type 2 diabetes mellitus. PLoS One 2012;7:e51236.

19. Boucher J, Masri B, Daviaud D, Gesta S, Guigné C, Mazzucotelli A, et al. Apelin, a newly identified adipokine up-regulated by insulin and obesity. Endocrinology 2005;146:1764-71. [CrossRef]

20. Bonnet F, Cooper ME. Potential influence of lipids in diabetic nephropathy: insights from experimental data and clinical studies. Diabetes Metab 2000;26:254-64.

21. Jacobs MJ, Kleisli T, Pio JR, Malik S, L'Italien GJ, Chen RS, et al. Prevalence and control of dyslipidemia among persons with diabetes in the United States. Diabetes Res Clin Pract 2005;70:263-9. [CrossRef]

22. Zhang BH, Wang W, Wang H, Yin J, Zeng XJ. Promoting effects of the adipokine, apelin, on diabetic nephropathy. PLoS One 2013;8:e60457.

23. Karakoç A, Sahin A, Polat ES, Aliyev E, Yildirim A, Bakan N, et al. Serum apelin and ADMA levels in type 2 diabetics with and without vascular complications. Diabetes Metab Syndr 2016;10:S106-9. [CrossRef]

24. Yonem A, Duran C, Unal M, Ipcioglu OM, Ozcan O. Plasma apelin and asymmetric dimethylarginine levels in type 2 diabetic patients with diabetic retinopathy. Diabetes Res Clin Pract 2009;84:219-23. [CrossRef] 